\title{
Assessment of Insulin Resistance and Serum Levels of Irisin in Patients with Idiopathic Infertility
}

A.A.Saleh ${ }^{1}$, A.M.Hamed ${ }^{2}$, A.A.Elfallah ${ }^{3}$ and E.M.Amin ${ }^{4}$

${ }^{1}$ Assistant Professor of Dermatology, Venereology and Andrology Dept., Faculty of Medicine, Benha Univ., Benha, Egypt

${ }^{2}$ Assistant Professor of Dermatology, Venereology and Andrology Dept., Faculty of Medicine, Benha Univ., Benha, Egypt

${ }^{3}$ Lecture of Clinical and Chemical Pathology Dept., Faculty of Medicine, Benha Univ., Benha, Egypt

${ }^{4}$ M.B.B.CH, Faculty of Medicine, Zagazig Univ., Zagazig, Egypt

E-Mail: dr_eman28@yahoo.com

\begin{abstract}
According to the World Health Organization (WHO), the definition of infertility is "the inability of a sexually active, noncontracepting couple to achieve spontaneous pregnancy in one year. The category 'unexplained male infertility' (UMI) is reserved for infertile men with infertility of unknown origin with normal semen and in which female infertility factors have been ruled out. Some cases of unexplained male infertility could be due to IR, leading to hypogonadism and other metabolic features. To evaluate serum level of irisin in patients with idiopathic infertility. Serum irisin concentrations were measured in 30 male patients with idiopathic infertility, between the ages of 25 and 50 years and 20 healthy controls using enzyme-linked immunosorbent assay (ELISA). Serum levels of irisin in male patients with idiopathic infertility were found to be lower than in healthy controls $(\mathrm{p}=0.001)$. Decreased serum irisin probably reflect the role of irisin in the diagnosis of idiopathic infertility.
\end{abstract}

Keywords: Idiopathic infertility, Irisin, ELISA.

\section{Introduction}

The World Health Organization defines infertility as the inability of a sexually active couple (at least three times per month), not using contraception, to achieve pregnancy within one year [1].

Many studies were done to reveal the potential relationship between metabolic syndrome (MetS) and male infertility aiming to provide new therapeutic options for infertile males [2].

Irisin is a newly discovered exercise-induced myokine suggested to induce browning in adipose tissue and thus increase energy expenditure. Irisin promotes fat burning and therefore has been suggested to improve metabolic and glucose homeostasis [3].

Elevated circulating (serum/plasma) irisin is associated with increased risk of metabolic syndrome and cardiovascular disease [4].

So decrease level of irisin may play a role in the pathogenesis of idiopathic infertility.

The aim of this study was to investigate the serum levels of irisin and insulin resistance in male patients with idiopathic infertility and their correlation to the disease.

\section{Patients and methods}

\subsection{Type of study}

This comparative case control study included 30 male patients with idiopathic infertility and 20 healthy, age and sex matched controls attending the dermatology outpatient clinic at Benha University Hospital, Benha, Egypt from September 2018 to March 2019. Participants gave their informed written consent before enrolment and the study was approved by the Research Ethics Committee in Faculty of Medicine, Benha University.

\subsection{All participants in this study were subjected to the following}

All patients included in the study was male patients suffer from idiopathic infertility between the ages of 25 and 50 years. Patients with varicocele, genetic infertility and hormonal abnormalities were excluded. None of the patients was on systemic or topical treatment for one month before the study.

\subsection{Laboratory investigations}

This test was done using Irisin and insulin ELISA Kits provided by CusabiolUSA according to the manufacturer's instructions.

\subsection{Measurement of human galectin-3}

Blood samples were obtained and samples were allowed to clot for two hours at room temperature before centrifugation for 15 minutes at $1000 \times \mathrm{g}$. We removed serum and did assay immediately and we stored samples at $-20^{\circ} \mathrm{C}$ or $-80^{\circ} \mathrm{C}$. we avoided repeated freeze-thaw cycles. After centrifugation, serum was separated by a pipette, divided and kept in eppendorf tubes labeled with the number of the person.

\subsection{Statistical analysis}

The statistical analysis was done using Statistical package for Social Science (IBM Corp. Released 2011. IBM SPSS Statistics for Windows, Version 20.0. Armonk, NY: IBM Corp.).

\section{Results}

This study included 50 subjects, 30 male patients with idiopathic infertility and 20 normal subjects as a control.

Mean serum level of irisin was statistically significant lower in patients than controls Table (1).

Insulin resistance values were higher in patients than controls denoting presence of insulin resistance in idiopathic infertility patients Table (2).

Table (1) Comparison between the two studied groups according to serum level of irisin 


\begin{tabular}{llllclll}
\hline & \multicolumn{2}{c}{ Patients $(\mathbf{n}=\mathbf{3 0})$} & \multicolumn{3}{c}{ Controls $(\mathbf{n}=\mathbf{2 0})$} & t & P \\
\hline Mean & $\mathbf{\pm}$ SD & Range & Mean & $\mathbf{\pm S D}$ & Range & & \\
\hline 2.92 & $2.98 \pm 0.61$ & $2.11-3.54$ & 10.06 & $9.96 \pm 2.29$ & $3.98-13.94$ & 15.12 & $<0.001$ (HS) \\
\hline
\end{tabular}

Table (2) Comparison between the two studied groups according to insulin resistance

\begin{tabular}{lccccccc}
\hline & \multicolumn{2}{c}{ Patients $(\mathbf{n}=\mathbf{3 0})$} & \multicolumn{3}{c}{ Controls $(\mathbf{n}=\mathbf{2 0})$} & $\mathbf{U}$ & P \\
\hline Mean & $\mathbf{\pm}$ SD & Range & Mean & $\mathbf{\pm S D}$ & Range & & \\
\hline 10.42 & $9.86 \pm 2.61$ & $2.28-15.94$ & 5.15 & $4.96 \pm 1.71$ & $0.98-9.07$ & 107.0 & $<0.001(\mathrm{~S})$ \\
\hline
\end{tabular}

\section{Discussion}

Unexplained or idiopathic male factor infertility means that there is no aetiological factor could be found using the common clinical, instrumental or laboratory methods [5]. Testicular histopathology of those patients shows various degrees of spermatogenic impairment but fail to identify specific pathogenesis [6].

The aim of this study was to evaluate the possible role of irisin and isulin resistance in the pathogenesis of idiopathic infertility in male patients by measuring thier level in the serum of the patients.

This study included 30 male patients with idiopathic infertility and 20 age and sex matched healthy control subject. These patients were chosen between ages 25-50 years and confirmed diagnoses by semen analysis and hormonal profile.

Results of the current study showed that serum irisin level was significantly lower in idiopathic infertility patients when compared to controls, which may be explained by presence of IR in idiopathic infertility patients, indicating that serum irisin can be used in diagnosis of idiopathic infertility and as marker for insulin resistance in infertile patients.

Irisin, which is involved in energy homeostasis, is a regulating factor of glucose metabolism. Increased levels of circulating irisin, which is cleaved from its precursor fibronectin type III domain-containing protein 5, were associated with improved glucose homeostasis by reducing insulin resistanc [3].

Results of the current study showed statistically higher values of HOMA IR among idiopathic infertility patients than controls.

Identification of idiopathic infertility in young patients should lead to awareness of an increased risk of insulin resistance and its consequences later in life for diseases. The result of the present study may raise awareness in patients with idiopathic infertility that lifestyle changes (e.g., weight control, exercise, and meals with a low glycemic index) in early life can reduce the risk of insulin resistance.

\section{Conclusion}

Our data add novel aspects to the role of irisin in the pathophysiology of idiopathic infertility in male patients. Also as the level of irisin decreased, the chance of the disease is increased.

\section{References}

[1] J. Farhi, A. Ben-Haroush, Distribution of causes of infertility in patients attending primary fertility clinics, Medical Clinics of North America, Vol.13 (1), PP.51-54, 2011.

[2] A. Agarwal, A. Majzoub, Role of antioxidants in male infertility, BJUI Knowledge, PP.1-9, 2016.

[3] S. Kumar, D. Agarwal, K. Sharma and T.R. Swain, Association of male infertility to metabolic syndrome and other related disorders, Journal of Integrative Nephrology and Andrology, Vol.2 (04), PP.107-116, 2015.

[4] P. Boström, J. Wu, M.P. Jedrychowski, A. Korde,LYe,J.C.LO,K.A.Boström,J.H.Choi,J.Z.Long ,S.Kajimura,M.C.Zingaretti and B.M.Spiegelman , A PGC1- $\alpha$-dependent myokine that drives brownfatlike development of white fat and thermogenesis, Journal of Integrative Nephrology and Andrology, Vol.8, PP.463-481, 2012.

[5] K.H. Park, L. Zaichenko, M. Brinkoetter,B. Thakkar,A.SahinEfe,K.E.Joung and M.A. Tsoukas, Circulating irisin in relation to insulin resistance and the metabolic syndrome, J Clin Endocrinol Metab, Vol.98(12), PP.4899-4907, 2013.

[6] G. Cavallini, Male idiopathic oligoasthenoteratozoospermia, Asian J Androl, Vol.(8), PP.143-157, 2006.

[7] D. Filipponi and R. Feil, Perturbation of genomic imprinting in oligozoospermia, Epub, Vol.4 (1), PP.27-30, 2009.

[8] Nieschlag, Eberhard, Behre, M. Hermann, Susan and Nieschlag, Male Reproductive Health and Dysfunction, International Journal of Andrology, Vol.33 (7), PP.34-46, 2010. 